\section{ITEM3: A general-purpose FORTRAN 10 test scoring and item analysis program}

\author{
RICHARD F. ANTONAK \\ University of New Hampshire, Durham, New Hampshire 03824
}

This program was developed and refined over a 3-year period following a review of published item analysis programs since 1970 and detailed critiques by computer users and consultants. ${ }^{1}$ The objectives for its development were that the program be: general purpose, yet comprehensive, to allow for user specified optional analyses, efficient in terms of run time, and easily employed by the widest possible audience of users. More specific details are available in ITEM3 User's Manual (available upon request).

Input. The job deck consists of the following cards: a run parameters card, a subtest specification card, title cards, a run options card, a subtest reorder key (optional), a data format card, answer key, data deck, item weights (optional), and a last card. The run parameters card specifies the number of subjects, subtests, and item response options, unit numbers for scratch files, and user preference for recoding subject data from alphanumeric to integer mode. The subtest specification card contains the number of items in each subtest (or total test). The title cards permit the user to describe the problem in a text which is reproduced on the output. The run options card contains user preference for: level of significance test of point-biserial coefficients, punched output, printed output, printed code book of subject scores, weighting of items, reordering of items into subtests, recoding of TRUE/ FALSE data values, printed matrices of subject by item responses, frequency distributions, missing data value, printing of phi coefficients, analyzing an external criterion score, and performing Cochran's $Q$ analysis. The optional subtest reorder key allows the user to construct subtests from the original test items if the items are not entered sequentially by subtest. The variable format card indicates the location of the correct alternative for each item on the following answer key and of the examinee identification number, criterion test score (if desired), and item responses on the subsequent data cards. The use of more than one card per examinee is permitted. The optional item weights are used in the computation of subject test scores for printed or punched output. If the user wishes to terminate the program, the last card must contain the word

The assistance of Mr. Kevin E. O'Grady is gratefully acknowledged. Portions of this program are based upon (with permission) his previous work (A program to compute point-biserial coefficients of correlation, Educational and Psychological Measurement, 1977, 37, 263-265).
DONE punched in columns 14 . On the other hand, if the user wishes to analyze another set of data, the last card must contain the word DATA punched in columns 14 and the second job deck follows, arranged sequentially (as described above) beginning with a run parameters card.

Output. The printed output includes:

(1) Titling information.

(2) Correct response key (recoded and/or reordered).

(3) Test statistics (mean, standard deviation, skewness, kurtosis).

(4) Item analysis. (a) Item indices-difficulty, variance, standard deviation, skewness, kurtosis, the number, percent, and mean test scores of those answering correctly and those answering incorrectly based on both total sample and total responding, and a frequency distribution of item difficulties. (b) Item decoy indices-number, percent, and test mean of those selecting each alternative (correct alternative marked) and those omitting the item, point-biserial r (both including and excluding the item score from the test score) tested against a user-specified level of significance for each alternative and for omits with significant coefficients marked.

(5) Test reliability (KR-20, standard error of measurement).

Optional output and features include:

(1) The analysis of items with from two [TRUE (1)/FALSE (2)] to seven response alternatives.

(2) Analyses for up to 10 subtests. (a) Subtest statistics, (b) subtest reliability, (c) subtest specificity (point-biserial $r$ of each item with each subtest checked for significance, phi coefficients, Cronbach's coefficient alpha).

(3) Reordering of test items into subtest according to a user-specified key.

(4) Recoding input data from alphanumeric to integer format to process machine-scored protocols (e.g., OPSCAN) and distinguish blanks from zeros.

(5) Input and analyses of an external criterion test score (criterion test statistics, test-criterion correlation, criterion-item analyses).

(6) Test, criterion, and subtest frequency distributions (raw score, grouped, and a histogram).

(7) Matrices of raw, recoded, reordered, and/or scored subject by item responses.

(8) Specification of a missing data value other than zero or blank.

(9) Recoding TRUE/FALSE data values if they are other than the default $(1 / 2)$ values.

(10) Specification of an alternate data input medium other than cards.

(11) Printed code book of subject scores in $8^{\frac{1}{2}} \mathrm{x}$ 11 in. format (ID, test, criterion, and subject raw, $z$, and standard scores). 
(12) Punched output of subject scores as detailed above.

(13) Weighting of individual test items for the computation of test scores for printed or punched output.

(14) Cochran's $Q$ analysis with associated $\mathrm{df}$ and exact probability value.

Restrictions. The program limitations impose a maximum of 150 items, 10 subtests, and 7 response alternatives plus omit. The upper limit of subjects is arbitrarily set at 9,999 , data with missing observations can be processed, and more than one deck can be analyzed at a time.
Availability. A copy of this paper, a program manual, and/or a source listing which includes data and printed output for sample problems may be obtained without charge from Dr. Richard F. Antonak, Education Department, Morrill Hall, University of New Hampshire, Durham, New Hampshire 03824. Those interested in obtaining the program are advised to communicate with Ms. Susan Sliwoski, Applications Librarian, Computer Services, Kingsbury Hall (603/862-2323), for cost and shipping information.

(Accepted for publication December 11, 1978.) 